\title{
Difficultés De Transport International De Marchandises: Proposition D'un Cadre D'étude; Cas Du Maroc
}

\section{Kawtar Akoudad}

Université Sidi Mohamed Ben Abdellah, Laboratoire de Productique, Énergétique et Développement Durable (LPE2D), Fès, Maroc

\section{Fouad Jawab}

Université Sidi Mohamed Ben Abdellah, Laboratoire de Productique, Énergétique et de Développement Durable LPE2D \& MIDLOG, Fès, Maroc

Doi: 10.19044/esj.2018.v14n14p137 URL:http://dx.doi.org/10.19044/esj.2018.v14n14p137

\begin{abstract}
This paper focuses on developing an approach that identifies the problems of the international freight transport in Morocco. It also arranges these problems in order to have a reliable and detailed picture of the transport state.

This problem was developed by approaching, in the first place, the importance and the role of the freight transport sector in Morocco. This sector, in addition to its vital importance for the economy, is of great social significance.

In the second place, we deployed the quality management tools (the Ishikawa diagram and QQQOQCP tool) in order to identify, through literature, the problems of the international freight transport and understand the fundamentals of each difficulty. This analysis was corroborated by surveys that gathered information, reformulated dissatisfaction, and discerned weaknesses of the transport.

In the last place and in order to prioritize the difficulties identified, we used the affinity diagram which allowed to model and group with clarity all the problems detected.
\end{abstract}

Keywords: Identification, difficulties, transport, freight, Morocco

Résumé

L'objectif de ce travail consiste à développer une démarche d'identification des problèmes que subit le transport international de marchandises au Maroc et ordonner ces problèmes en vue d'avoir une vision fiable et fine sur l'état du transport. 
Nous avons développé cette problématique en approchant, dans un premier lieu, l'importance et le rôle du secteur de transport de marchandise au Maroc. En effet, ce secteur, en plus de son importance capitale pour l'économie, est d'une grande portée sociale.

En deuxième lieu, nous avons déployé les outils de management de la qualité (le diagramme d'Ishikawa et l'outil QQQOQCP) afin de pouvoir identifier, via l'analyse documentaire, les problèmes que subit le transport international de marchandises et comprendre les fondements de chaque difficulté. Cette analyse a été corroborée par des enquêtes qui ont permis de rassembler les informations, reformuler les insatisfactions et discerner les faiblesses du transport.

En dernier lieu, nous avons eu recours au diagramme des affinités qui a permis de modéliser et regrouper avec clarté l'ensemble des problèmes décelés.

Mots-clés: Identification, difficultés, transport, marchandises, Maroc

\section{Introduction}

Par route, par mer, par voie ferroviaire ou aérienne, le secteur du transport des marchandises est capital dans le développement économique et social. Étant au cœur des différents secteurs stratégiques, le fret représente un levier d'amélioration de la compétitivité des échanges commerciaux du Maroc aussi bien intérieurs qu'extérieurs et une aubaine pour la création d'emploi (PNAUD, 2015). Le développement des réseaux commerciaux crée davantage des besoins de gestion de transport et nécessite la mise en place de services performants répondant aux contraintes de temps, de fiabilité et de qualité. Ces besoins se sont accentués au Maroc par l'ouverture commerciale, entamée par le pays au milieu des années 80, et la conclusion d'Accords de libre échange, au niveau bilatéral ou régional, au cours de la dernière décennie.

Néanmoins, ce secteur pâtit de plusieurs difficultés qui freinent son développement et entravent son fonctionnement. Le cas du Maroc soulevé dans cet article sera traité dans l'objectif de mettre en évidence les problèmes dont souffre ce secteur tout en se limitant au transport international de marchandises.

Ainsi, nous avons abordé le sujet en évoquant, dans un premier lieu, l'importance et le rôle du secteur de transport de marchandises au Maroc. En effet, ce secteur est indéniablement nécessaire pour le bon fonctionnement du tissu productif de par sa présence au niveau de tous les maillons de la chaîne de valeur, de l'amont à l'aval. Sans oublier qu'il participe à la création d'emploi et dans le désenclavement des zones reculées du territoire national ce qui permet de juguler les pressions monopolistiques et les inégalités interrégionales. 
En un deuxième lieu, nous avons déployé des outils de management de la qualité à savoir le diagramme d'Ishikawa qui permet de structurer à travers ces 7M (Matières, Milieu, Méthode, Matériels, Main d'œuvre, Management, Moyen financier) les composantes du transport international de marchandises, ainsi que l'outil QQQOQCP qui permet de formaliser le descriptif d'un problème en se posant des questions élémentaires (Qui ? Quoi ? Où ? Quand ? Comment ? Pourquoi ? Combien ?), au niveau de chaque composante classée par la méthode Ishikawa.

En un troisième lieu, nous avons soumis la liste des problèmes obtenue de l'analyse documentaire aux jugements des enquêtés via la méthode Delphi. Ce qui nous a permis de rassembler les informations, reformuler les insatisfactions et discerner les faiblesses du transport.

En dernier lieu et dans l'intention de structurer les problèmes détectés, nous avons fait appel au diagramme des affinités qui a permis de modéliser et regrouper avec clarté l'ensemble des problèmes.

\section{Importance et rôle du secteur de transport de marchandises au Maroc}

Le secteur du transport de marchandises est un secteur porteur pour l'économie nationale avec 100.000 emplois directs et une contribution de 5\% au PIB pour l'ensemble de la filière logistique dont 3\% pour le chargement et le transport (Kammas, 2015). L'importance du secteur de transport de marchandises se mesure également par son impact direct sur la compétitivité du tissu économique aussi bien en termes d'export que d'import.

Aussi il faut noter que le secteur de transport contribue, à lui seul, à hauteur de 5,8\% du total des valeurs ajoutées et $10,3 \%$ de celle des activités tertiaires en 2011 aux prix constants. Sa valeur ajoutée est passée de 19,6 milliards de dirhams (+/- 1,71 milliards d'euro) en 2000 à 33,8 milliards de dirhams (+/- 2,94 milliards d'euro) en 2011, soit une croissance annuelle moyenne de 5\%. En termes d'emplois créés, le transport (y compris entrepôts et communications) a employé, en 2011, près de 493.460 personnes ce qui représente $4,7 \%$ de la population active occupée, dont $76 \%$ dans le milieu urbain (Van Wulpen, 2014). En outre, le transport, et en particulier celui des marchandises, est indéniablement nécessaire pour le bon fonctionnement du tissu productif de par sa présence au niveau de tous les maillons de la chaîne de valeur, de l'amont à l'aval (Kammas, 2015). Il s'agit, de ce fait, d'un secteur d'appui contribuant à la croissance nationale, comme en témoigne la corrélation positive entre l'évolution de l'activité économique globale et la croissance du secteur. De même, le rôle du secteur du transport est capital dans l'intégration de l'économie nationale dans l'économie mondiale ainsi que dans le désenclavement des zones reculées du territoire national permettant de juguler les pressions monopolistiques et les inégalités interrégionales. 


\section{Démarche d'identification des problèmes du TIM au Maroc}

L'intérêt d'avoir une méthodologie pour analyser les problèmes est d'utiliser un processus avec un maximum d'exhaustivité. La méthodologie assortie d'outils, permet de prendre en considération la complexité des situations et d'approcher les causes fondamentales d'un dysfonctionnement afin de pouvoir proposer des solutions efficaces et définitives.

Comme nous venons de souligner, l'analyse d'un problème revient à s'appuyer sur un grand nombre d'outils existants. La démarche qualité propose toute une panoplie d'outils qui permettent de répondre à cette requête (Connie, 2009). Il convient certes de maîtriser les outils que nous utilisons, mais aussi de savoir lequel choisir selon la problématique à traiter et selon l'étape où nous nous situons, car une fois encore, la pertinence est de mise. Ainsi, nous avons mené en premier lieu une comparaison entre plusieurs outils de qualité (voir Tableau 1) ce qui nous a permis de designer les plus adéquats à notre étude.

Tableau 1. Récapitulatif des méthodes de résolution des problèmes

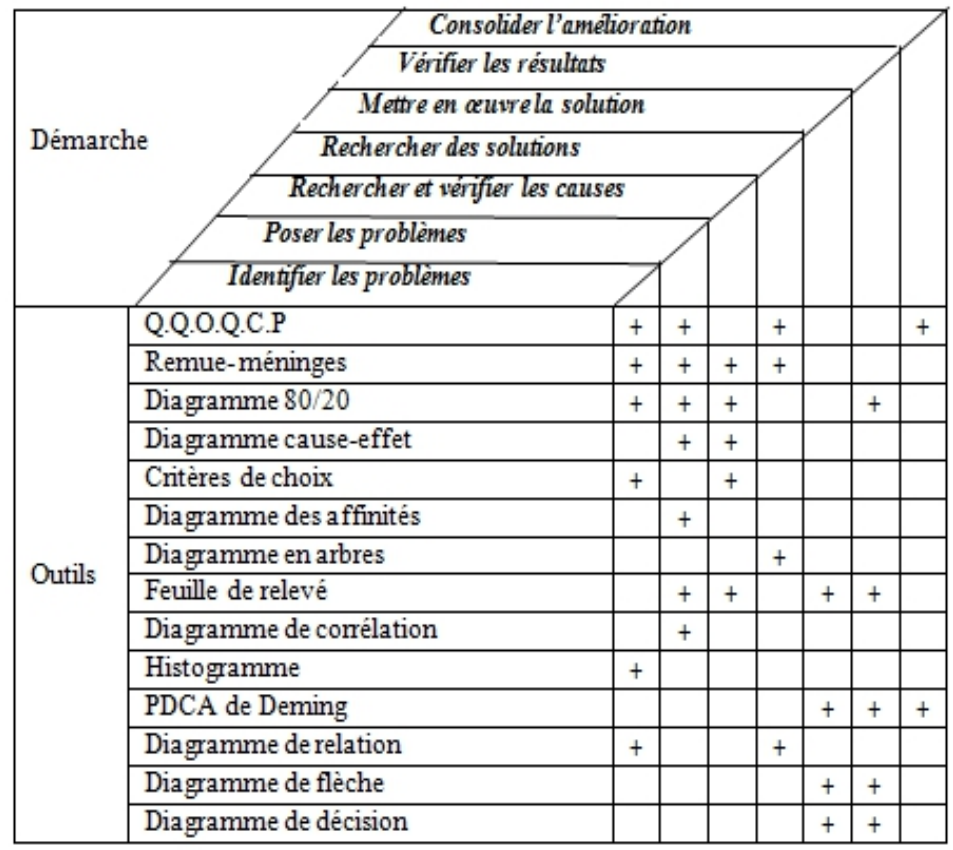

Ensuite nous avons implémenté ces outils au niveau de notre démarche d'identification et d'hiérarchisation des problèmes (voir Figure 1). 

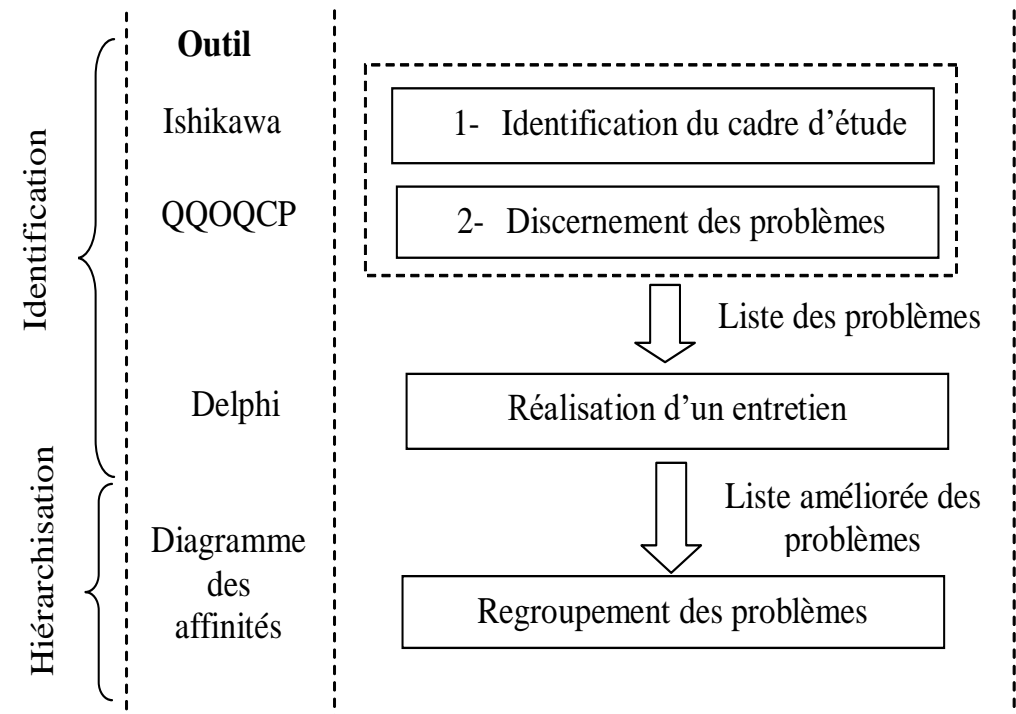

\section{Etape}

Analyse documentaire

Recueil des perceptions

Représentation: des problèmes

Figure 1. Architecture générale de la méthodologie d'identification et d'hiérarchisation des problèmes du TIM au Maroc

En se basant sur le tableau comparatif notre choix a reposé sur 3 outils. Ainsi pour mener l'analyse documentaire et plus précisément définir le cadre d'étude, nous avons opté pour le diagramme d'Ishikawa car il permet de prendre en considération les différentes composantes du TIM (matérielles, de gestion, financières, etc....). Le QQOQCP s'est avéré l'outil le plus adéquat pour identifier les problèmes au niveau de chaque composante du TIM lors de l'analyse documentaire. Le résultat de cette analyse est ensuite consolidé par un recueil des perceptions des parties prenantes du TIM. Ainsi nous avons reformulé la liste des problèmes obtenue puis soumis cette liste au jugement des enquêtés. La méthode adoptée pour effectuer cette opération est la méthode Delphi. Finalement pour modéliser et regrouper avec clarté les problèmes enrichis (issus des entretiens), nous avons fait appel au diagramme des affinités qui est un puissant outil de structuration des données non chiffrées.

\section{Analyse documentaire \\ Définition du cadre d'étude}

Vue la complexité du TIM qui articule plusieurs dimensions et composantes (sociale, humaine, économique, environnementale, institutionnelle, etc.), il s'est avéré nécessaire de commencer tout d'abord par esquisser un cadrage de l'aire d'étude. Ainsi nous avons emprunté et adapté, à partir du domaine industriel, un outil qui englobe l'ensemble de ces composantes; il s'agit bien du diagramme d'Ishikawa. Cet outil permet une représentation structurée de toutes les constitutions du TIM en les catégorisant 
selon la règle des 7M (Milieu, Matière, Matériel, Main-d'œuvre, Management, Méthode, Moyens financiers). La représentation graphique du diagramme prend la forme schématique d'une arête de poisson. On trace donc, dans un premier temps, une flèche horizontale avec, à l'extrémité, l'expression de la composante principale qui est dans notre cas le TIM. Les arêtes qui convergent vers la ligne centrale, correspondent aux 7M. Chacune des 7M comprend des extensions qui représentent les composantes du TIM (voir Figure 2).

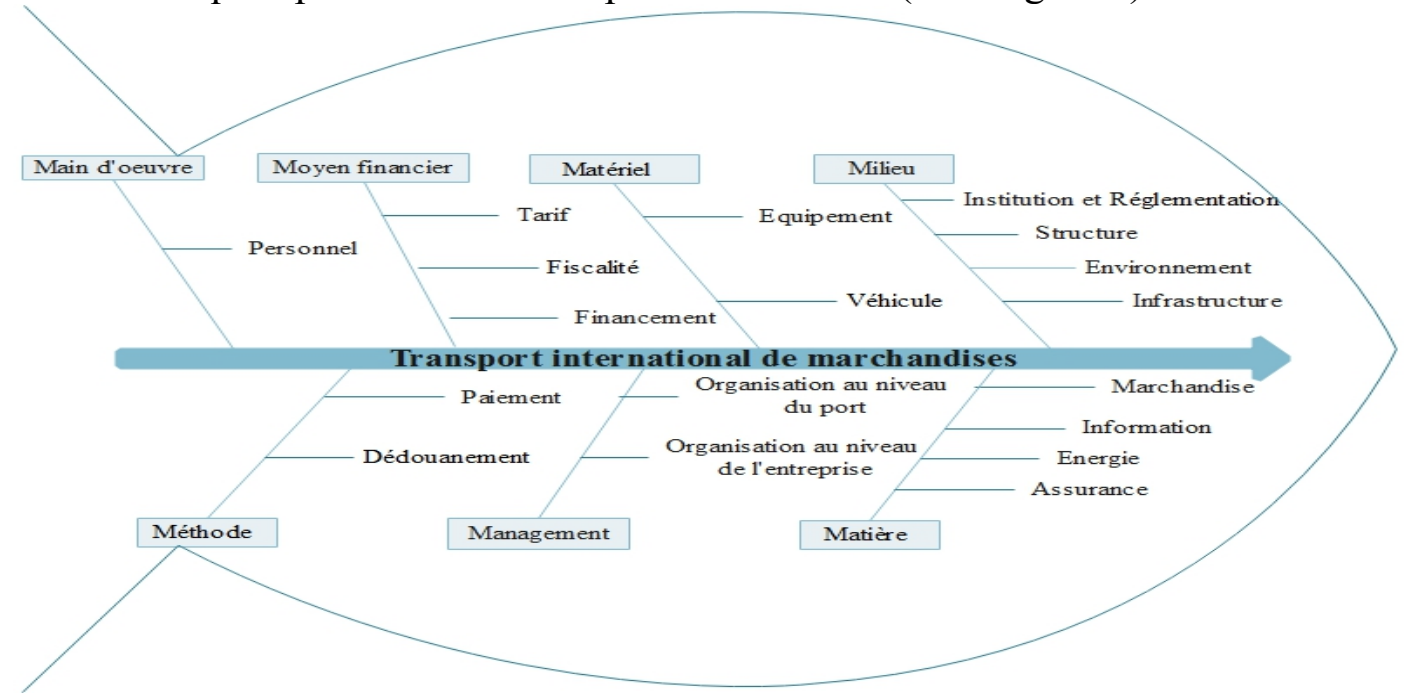

Figure 2. Diagramme d'Ishikawa du TIM

\section{Discernement des problèmes}

Pour identifier les problèmes au niveau de chacune des composantes du diagramme Ishikawa, nous avons opté pour l'outil QQOQCP qui adopte une démarche d'analyse critique constructive basée sur le questionnement systématique (Akoudad \& Jawab, 2018) (voir Tableau 2).

Tableau 2. Composantes de l'outil QQOQCP

\begin{tabular}{|c|c|c|}
\hline Questions & Exemple des questions à poser & Exemple des cibles \\
\hline $\begin{array}{c}\text { Quoi? permet une meilleure } \\
\text { description du problème }\end{array}$ & $\begin{array}{c}\text { De Quoi s'agit-il ? } \\
\text { Quelles sont les caractéristiques? } \\
\text { Quelles sont les conséquences? }\end{array}$ & $\begin{array}{c}\text { les actions } \\
\text { les objets } \\
\text { les méthodes } \\
\text { les opérations }\end{array}$ \\
\hline $\begin{array}{c}\text { Qui? permet une meilleure } \\
\text { description des acteurs ou } \\
\text { personnes concernées }\end{array}$ & $\begin{array}{c}\text { Qui est concerné ? } \\
\text { Qui a le problème? } \\
\text { Qui est interessé par le résultat? }\end{array}$ & $\begin{array}{c}\text { Responsable, victime, acteur } \\
\text { Clients, opérateurs, } \\
\text { Compétence, qualification,... }\end{array}$ \\
\hline $\begin{array}{c}\text { Où? permet la description des } \\
\text { lieux }\end{array}$ & $\begin{array}{c}\text { Où cela se produit-il? } \\
\text { Dans quel lieu? }\end{array}$ & $\begin{array}{c}\text { Lieux, local, distance ... } \\
\text { Service, ... }\end{array}$ \\
\hline $\begin{array}{c}\text { Quand? permet de bien définir } \\
\text { les temps }\end{array}$ & $\begin{array}{c}\text { Quand cela apparaît-il ? } \\
\text { Quelle est sa fréquence? ? } \\
\text { Quand se produit le risque ? }\end{array}$ & $\begin{array}{c}\text { Mois, jour, heure, } \\
\text { Moments, périodicité, } \\
\text { fréquence, prévisibilité }\end{array}$ \\
\hline $\begin{array}{c}\text { Comment? permet une } \\
\text { meilleure description de la } \\
\text { manière ou de la méthode }\end{array}$ & $\begin{array}{c}\text { Comment se produit le problème? } \\
\text { Dans quelles conditions ou } \\
\text { circonstances? }\end{array}$ & $\begin{array}{c}\text { Méthode, modes opératoires, } \\
\text { Organisation du travail. } \\
\text { Procédures, règlements, } \\
\text { consignes. }\end{array}$ \\
\hline
\end{tabular}




\begin{tabular}{|c|c|c|}
\hline & $\begin{array}{c}\text { Avec quelles méthodes, quels } \\
\text { moyens? }\end{array}$ & $\begin{array}{c}\text { Equipements, matière } \\
\text { première. }\end{array}$ \\
\hline $\begin{array}{c}\text { Pourquoi? peut se poser à la } \\
\text { suite des autres questions. }\end{array}$ & $\begin{array}{c}\text { Pourquoi ? à chaque réponse des } \\
\text { questions précédentes }\end{array}$ & $\begin{array}{c}\text { Toutes les cibles concernant } \\
\text { les autres questions. }\end{array}$ \\
\hline
\end{tabular}

Cet outil était notre guide lors de l'analyse documentaire des données disponibles. Il nous a permis de déceler un ensemble de problèmes par lesquels traverse le TIM sur plusieurs niveaux en répondant à chacune des questions constituant cette méthode.

Apres avoir obtenu la liste des problèmes, nous avons jugé que l'analyse documentaire, à elle seule, est insuffisante et qu'il est nécessaire de recueillir les perceptions des parties prenantes du TIM afin d'enrichir, vérifier et consolider cette liste.

\section{Enquête qualitative via la méthode Delphi Choix et définition de la méthode}

La méthode de travail devait répondre à un certain nombre d'exigences contradictoires, soit la disponibilité d'un seul enquêteur et un temps d'enquête limité; la nécessité de s'adresser à un public large afin de considérer toutes les préoccupations individuelles comme collectives et l'objectif d'obtenir une vision riche et diversifiée (Clément \& Madec, 2006). Cette contradiction était une des raisons qui plaidaient en faveur du choix de la méthode Delphi qui se distingue, selon (Booto Ekionea et al., 2011), des techniques usuelles de communication de groupe sur plusieurs plans. De cette manière, la méthode aide à consigner les opinions d'experts dans un domaine précis, limite le nombre de participants à l'étude (entre 7 et 18 experts) (Paliwoda, 1983, cité par Okoli \& Pawlowski, 2004) facilitant du même coup le travail d'identification et de sélection d'experts et offre une flexibilité lors de la conception et l'administration du questionnaire. Cette méthode se compose d'une série d'interrogations répétées, habituellement au moyen de questionnaires, d'un groupe d'individus dont les avis ou les jugements sont d'intérêt. Après l'interrogation initiale de chaque individu, chaque interrogation suivante est accompagnée de l'information concernant les réponses du tour précédent. L'individu est ainsi encouragé à reconsidérer et, si approprié, à changer sa réponse précédente à la lumière des réponses des autres membres du groupe (IIASA, 2017). Il s'agit donc d'une méthode visant à organiser la consultation d'experts sur un sujet précis.

\section{Mise en œuvre de la méthode}

L'étude que nous avons menée, a fait appel à une méthode Delphi réalisée en deux étapes afin de solliciter les opinions d'experts vis à vis du TIM. En effet, assez souvent dans les études Delphi, les améliorations ont lieu entre les premiers et deuxièmes tours d'évaluation, des itérations 
supplémentaires n'accroissent pas de façon significative la convergence des résultats (Chtioui, 2010).

Il faut noter que la validité des résultats d'un Delphi dépend du savoir, de la compétence et surtout de la coopération intentionnelle des experts consultés. D’où la nécessité de sélectionner des experts en fonction de leur expérience et de leur familiarité avec l'objet d'étude. Vu la multitude des parties prenantes du TIM, nous avons choisi pour cette étude un panel d'experts, constitué de commissionnaires de transport international car ces derniers, responsables de l'organisation du transport, traitent avec l'ensemble de ses acteurs et donc ils sont les mieux placés pour identifier ses problèmes. Pour la taille du panel à consulter, aucune règle stricte n'existe dans la littérature. Elle peut varier d'un petit nombre d'experts (Pelet, 2011) à des centaines (Landeta, 2006). (Vernette, 1985) considère que le seuil minimal du nombre d'experts est de 5 à 7 et qu'une dizaine d'experts Delphi constitue le meilleur rapport précision/coûts. Dans notre cas nous avons, vu le temps limité, consulté 5 personnes.

Lors de la première phase de l'étude, nous avons essayé d'élaborer une liste de questions ouvertes concernant les problèmes rencontrés par les experts et qui sont liés à l'ensemble des composantes du transport (7M). Les questions sont énoncées de façon à amener les expert à se positionner et argumenter leurs réponses. Nous avons préféré pour nos consultations, des entretiens en face à face ce qui nous a offert la possibilité de discuter avec les experts et de s'assurer qu'ils ont bien compris le sens des différentes questions avant de donner leurs avis.

Les réponses concernant les problèmes du TIM ont été compilées, examinées et classées selon la fréquence d'apparition du problème puis enrichies par la liste des problèmes obtenue via l'analyse documentaire que nous avons déjà établie ce qui a permis d'élaborer le deuxième questionnaire.

Lors de la deuxième phase, nous avons administré aux experts un nouveau questionnaire qui est le résultat enrichi de la première phase. Ainsi nous avons demandé à chaque expert d'exprimer sont point de vu à l'égard des problèmes, et de commenter leurs réponses s'ils le souhaitent.

\section{Hiérarchisation des problèmes du TIM au Maroc}

Afin d'hiérarchiser les problèmes obtenus à partir de l'étape précédente, nous avons eu recours au diagramme des affinités qui est un puissant outil de structuration des données et une méthode simple pour modéliser et regrouper avec clarté les problèmes complexes tout en mettant en évidence les liens entre eux. Cet outil est aussi connu sous le nom de 'Méthode KJ' venant de son auteur Kawakita Jiro (1964). Plusieurs étapes sont nécessaires pour la réalisation de ce diagramme à savoir : 
i) Faire la collecte des problèmes se rapportant au TIM (voir partie Identification).

ii) Relire les problèmes et essayer de découvrir ceux qui ont des points communs.

iii) Regrouper les problèmes qui présentent des similitudes et clarifier le contenu de chaque problème lorsqu'il est nécessaire.

iv) Identifier chaque catégorie (en prenant en considération les composantes du diagramme Ishikawa) avec une description sommaire des problèmes.

v) Répéter les étapes 3 à 5 jusqu'à une convergence.

vi) Tracer le diagramme final

\section{Résultats}

Après avoir listé les composantes qui constituent le TIM et une fois soumises à la méthode QQOQCP et la méthode Delphi, nous avons obtenu une longue liste des problèmes que nous avons structurés à l'aide du diagramme des affinités.

\section{Modèle 7M pour la représentation des difficultés du TIM}

Le modèle 7M (voir figure 4) issue de la démarche que nous avons développée, représente sous forme d'arbre l'ensemble des problèmes se situant au premier niveau du tableau obtenu (voir figure 5). Ce niveau résultat du regroupement selon plusieurs affinités (par exemple Milieu, Infrastructure, Institution et Réglementation ; Structure, et Environnement), se décline à son tour en un niveau inferieur, donnant lieu à des sous problèmes (exemple du problème $\mathrm{P}_{6}$ qui se décline en 7 sous problèmes). 


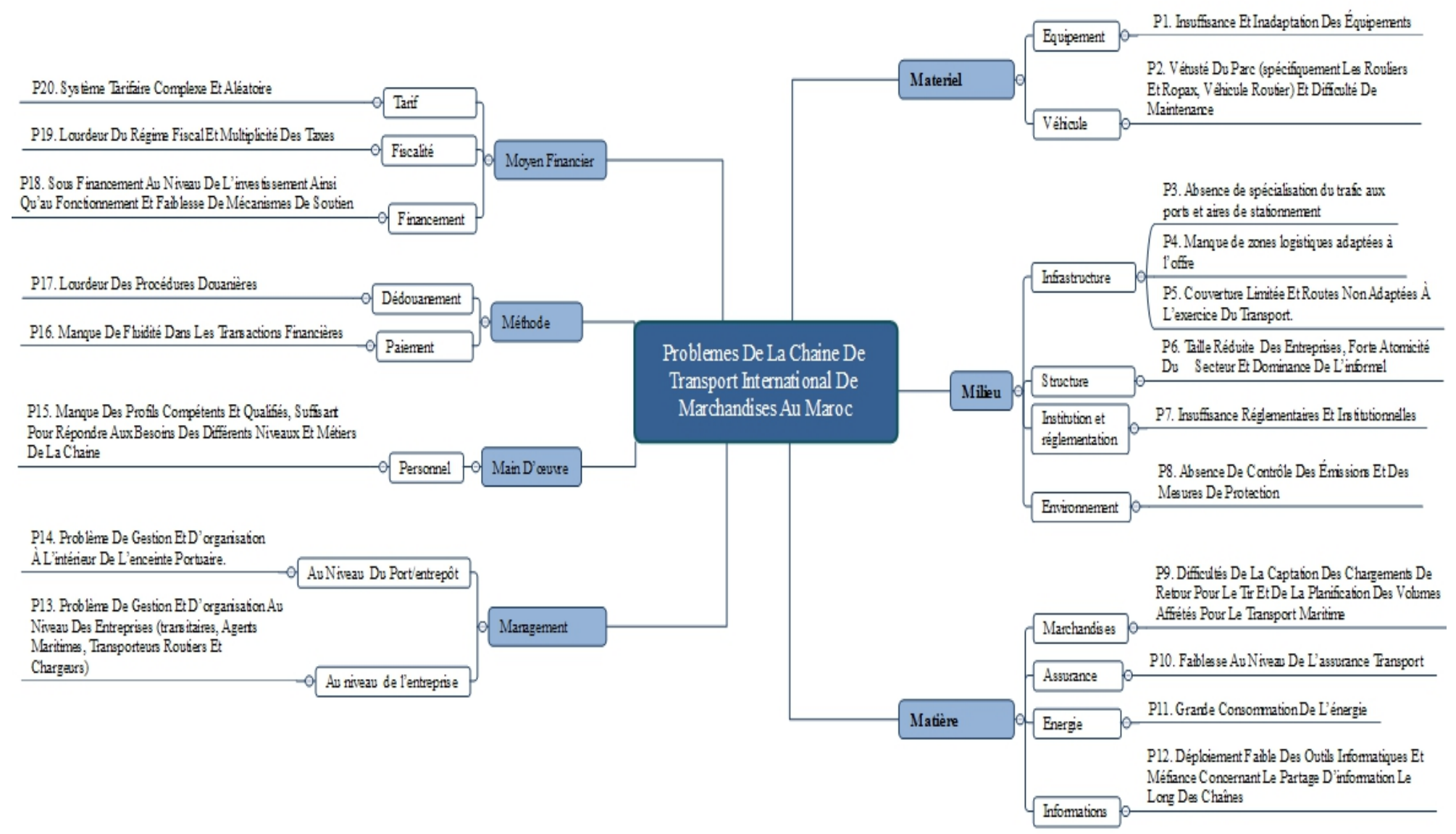

Figure 4. Modèle représentatif des problèmes de TIM 


\section{Interprétation}

Prenons en considération l'exemple donné par la Figure 5 qui traite l'aspect milieu du TIM, plus précisément la structure du marché. Ce regroupement comprend au premier niveau un seul problème P6 (Taille réduite des entreprises, forte atomicité du secteur de transport et dominance de l'informel) qui rassemble plusieurs sous problèmes $\mathrm{P}_{6,1}$ (Atomisation de l'offre de transport) ; $\mathrm{P}_{6,2}$ (Prédominance du secteur informel) ; $\mathrm{P}_{6,3}$ (Taille réduites des entreprises de transport); $\mathrm{P}_{6,4}$ (Faible développement des prestataires logistiques); $\mathrm{P}_{6,5}$ (Structures non adaptées des entreprises à la logique logistique) ; $\mathrm{P}_{6,6}$ (Présence limitée de fournisseurs tiers de services logistiques); $\mathrm{P}_{6,7}$ (Faible taille de nombreux chargeurs qui ne peuvent supporter le coût de l'externalisation).

Ainsi, le transport routier des marchandises pâtit de plusieurs difficultés. Parmi celles-ci, il y a lieu de citer, l'atomicité et la fragmentation qui se reflètent dans la composition du secteur dominée par les TPE et les PME qui représentent $95 \%$ des entreprises opérant dans le secteur dont $90 \%$ sont des entreprises individuelles, qui détiennent chacune une flotte moyenne de 3 à 5 camions. Ces petites entités échappent généralement à la réglementation sociale et fiscale face aux entreprises structurées qui ne représentent que $10 \%$. De même, la forte concurrence exercée par l'informel, dont la part dépasse $40 \%$, constitue un véritable handicap au développement du secteur (AMDL, 2016). L'ensemble de ces facteurs contribue à rendre le coût du transport au Maroc relativement élevé et impacte négativement la compétitivité de la production nationale.

Quant au transport maritime, qui assure plus de 95\% du transport des échanges extérieurs, il se heurte à un certain nombre d'obstacles relatifs, notamment, à la participation de la flotte marocaine dans le transport. Ainsi, après la faillite de la compagnie Comarit/Comanav Ferry en 2012, l'IMTC group, important armateur marocain privé avec 7 porte-conteneurs, 2 rouliers, 2 car-ferries jusqu'à une date récente, a cédé 3 porte-conteneurs à l'armateur allemand Lloyds List en 2013. Par conséquent, la participation du pavillon marocain au commerce extérieur demeure limitée par rapport à celle des armateurs étrangers qui s'en accaparent (93\%) en 2012, en raison, notamment, de l'absence totale des armateurs marocains sur le segment du trafic de vrac qui représente la majorité du trafic maritime (77\%) (DEPF, 2016).

Le faible développement des prestataires logistiques et le manque de sensibilisation à l'importance de la logistique parmi les entreprises marocaines ne contribuent pas à l'émergence de ces services. Seulement une poignée d'entreprises propose des services logistiques liés au transport. Les entreprises qui offrent une palette complète de services logistiques sont quasiment exclusives aux filiales de groupes européens et elles ont, le plus souvent, comme clients des entreprises multinationales. De même, l'offre de conseil en 
logistique est quasiment inexistante. Exel est entré sur ce marché mais la demande reste faible. Les entreprises marocaines ont généralement peu intégré la démarche « Supply Chain Management », c'est-à-dire la gestion logistique optimisée, considérée comme un facteur important de compétitivité. Aussi, les professionnels du métier relèvent que la méfiance des chargeurs marocains pour une transparence sur leurs stocks, leurs cadences de production etc.... est un frein important au développement des services logistiques. De même, la petite taille de nombreux chargeurs explique pourquoi l'externalisation logistique peine à émerger. La réduction des coûts est un des avantages recherchés. Cette réduction est visible à partir du moment où l'entreprise a une idée exacte de ces coûts cachés. Pour la majorité des entreprises marocaines, cette information n'est pas disponible et les impératifs de flexibilité et d'amélioration du service ne sont pas intégrés. La faiblesse des services connexes à la logistique est patente. Ce fait est corroboré par la faible activité dans ce domaine. Cela a des conséquences importantes pour le développement de ce qui fait partie intégrante d'une logistique moderne, le développement de plates-formes logistiques (MET, 2006).

\section{Conclusion}

Le secteur du transport, notamment dans sa composante marchandise, revêt une importance majeure dans la dynamique économique. Son importance découle de sa contribution au fonctionnement des marchés des produits et de facteurs de productions, d'une part, et de son apport en matière de l'amélioration des grandeurs macroéconomiques d'autre part (DEPF, 2013).

Ce secteur, représenté en terme réduit en sa version internationale, malgré son importance éprouve plusieurs difficultés sur plusieurs niveaux, un constat qui a été confirmé par l'analyse documentaire et le recueil de perception des parties prenantes que nous avons mené. Les principaux blocages que nous avons pu ressortir sont liés à l'organisation de la profession, relative à l'insuffisance de l'infrastructure pour certaines de ses composantes, et au retard en matière des services logistiques. Les actions entreprises par les pouvoirs publics doivent s'inscrire ainsi dans l'objectif de moderniser et de mettre à niveau le secteur mentionné, à travers l'amélioration de l'organisation et du fonctionnement de la profession en vue d'en améliorer la concurrence et la compétitivité, le développement de l'infrastructure et l'encouragement des reformes en matière de service logistique. 


\begin{tabular}{|c|c|c|c|}
\hline & & \multirow[b]{2}{*}{ Niveau.1 } & \multirow[b]{2}{*}{ Niveau.2 } \\
\hline & & & \\
\hline \multirow[t]{2}{*}{ Matériel } & Equipement & $\mathrm{P}_{1}$. Insuffisance et inadaptation des équipements & $\begin{array}{l}\mathrm{P} 1,1 \\
\mathrm{P} 1, \mathrm{n} \\
\end{array}$ \\
\hline & Véhicule & $\begin{array}{l}\mathrm{P}_{2} \text {. Vétusté du parc (spécifiquement les Rouliers et } \\
\text { RoPax, véhicule routier) et difficulté de maintenance }\end{array}$ & \\
\hline \multirow[t]{6}{*}{ Milieu } & Infrastructure & $\begin{array}{c}\mathrm{P}_{3} \text {. Absence de spécialisation du trafic aux ports et } \\
\text { d'aires de stationnement }\end{array}$ & \\
\hline & & $\mathrm{P}_{4}$. Manque de zones logistiques adaptées à l'offre & \\
\hline & & $\begin{array}{l}\mathrm{P}_{5} \text {. Couverture limitée et routes non adaptées à l'exercice } \\
\text { du transport. }\end{array}$ & I \\
\hline & Structure & $\begin{array}{l}\mathrm{P}_{6} . \text { Taille réduite des entreprises, forte atomicité du } \\
\text { secteur de transport et dominance de l'informel }\end{array}$ & \\
\hline & $\begin{array}{l}\text { Institution et } \\
\text { réglementation }\end{array}$ & $\mathrm{P}_{7}$. Insuffisance réglementaires et institutionnelles & \\
\hline & Environnement & $\begin{array}{l}\mathrm{P}_{8} \text {. Absence de contrôle des émissions et des mesures de } \\
\text { protection }\end{array}$ & \\
\hline \multirow[t]{4}{*}{ Matière } & Marchandises & $\begin{array}{l}\mathrm{P}_{9} \text {. Difficultés de la captation des chargements de retour } \\
\text { pour le TIR et de la planification des volumes affrétés } \\
\text { pour le transport maritime }\end{array}$ & \\
\hline & Assurance & $\mathrm{P}_{10}$. Faiblesse au niveau de l'assurance transport & \\
\hline & Energie & $\mathrm{P}_{11}$. Grande consommation de l'énergie & \\
\hline & Informations & $\begin{array}{c}\mathrm{P}_{12} \text {. Déploiement faible des outils informatiques et } \\
\text { méfiance concernant le partage d'information le long des } \\
\text { chaînes }\end{array}$ & \\
\hline \multirow[t]{2}{*}{ Management } & $\begin{array}{l}\text { Au niveau de } \\
\text { l'entreprise }\end{array}$ & $\begin{array}{c}\mathrm{P}_{13} \text {. Problème de gestion et d'organisation au niveau des } \\
\text { entreprises (transitaires, agents maritimes, transporteurs } \\
\text { routiers et chargeurs) }\end{array}$ & \\
\hline & $\begin{array}{c}\text { Au niveau du } \\
\text { port/entrepôt }\end{array}$ & $\begin{array}{l}\mathrm{P}_{14} \text {. Problème de gestion et d'organisation à l'intérieur de } \\
\text { l'enceinte portuaire. }\end{array}$ & \\
\hline Main d'œuvre & Personnel & $\begin{array}{l}\mathrm{P}_{15} \text {. Manque des profils compétents et qualifiés, suffisant } \\
\text { pour répondre aux besoins des différents niveaux et } \\
\text { métiers de la chaine }\end{array}$ & \\
\hline \multirow[t]{2}{*}{ Méthode } & Paiement & $\mathrm{P}_{16 .}$ Manque de fluidité dans les transactions financières & \\
\hline & Dédouanement & $\mathrm{P}_{17 .}$ Lourdeur des procédures douanières & \\
\hline \multirow[t]{3}{*}{ Moyen financier } & Financement & $\begin{array}{l}\mathrm{P}_{18} \text {. Sous financement au niveau de l'investissement } \\
\text { ainsi qu'au fonctionnement et faiblesse de mécanismes } \\
\text { de soutien. }\end{array}$ & \\
\hline & Fiscalité & $\mathrm{P}_{19}$. Lourdeur du régime fiscal et multiplicité des taxes & \\
\hline & Tarif & $\mathrm{P}_{20}$. Système tarifaire complexe et aléatoire & $\begin{array}{l}\mathrm{P} 20,1 \\
\mathrm{P} 20, \mathrm{n}\end{array}$ \\
\hline
\end{tabular}

Figure 5. Exemple d'opérationnalisation des difficultés du transport international de marchandises au Maroc

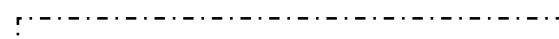
- $\mathrm{P}_{6,1}$. Atomisation de l'offre de transport. En effet, 90\% des entreprises possèdent 1 ou 2 camions et seulement 50 entreprises possèdent une flotte de plus de 20 camions ;

- $\mathrm{P}_{6,2}$. Prédominance du secteur informel, estimé à $70-75 \%$ du transport (routier) annuel total

'• $\mathrm{P}_{6,3}$. Taille réduites des entreprises de transport

¿ $\mathrm{P}_{64}$. Faible développement des prestataires logistiques (manque de sensibilisation de leurs importances)

- $\mathrm{P}_{6,5}$. Structures non adaptées des entreprises à la logique logistique (structures pyramidales, cloisonnement des fonctions, rétention i l'information, etc.).

- $P_{6,6}$. Présence limitée de fournisseurs tiers de services logistiques,

• P67. Faible taille de nombreux chargeurs qui ne peuvent supporter le coût de l'externalisation. 


\section{References:}

1. Akoudad, K, et Jawab, F., 2018, Road Transport Problems in Morocco: Discernment and Classification, Proceedings of the International Conference on Industrial Engineering and Operations Management Bandung, Indonesia.

2. AMDL (Agence marocaine de développement logistique), 2016, Logistique au Maroc, acquis importants, grands défis et perspectives prometteuses.

3. Booto Ekionea, J.-P., Bernard, P., et Plaisent, M., 2011, Consensus par la méthode Delphi sur les concepts clés des capacités organisationnelles spécifiques de la gestion des connaissances, Université du Québec à Montréal. Recherches Qualitatives, Vol. 29, No.3, pp. 168-192.

4. Chtioui, T., 2007, Les fondements d'un modèle de mesure de la communication pour le contrôle de gestion : une étude Delphi, Comptabilité et environnement, <halshs-00544890>.

5. Clément, O., et Madec, P., 2006, Un outil pour la construction d'indicateurs de développement durable : la méthode Delphi. Une expérience en aquaculture, Natures Sciences Societies, Vol. 14, pp. 297-302.

6. Connie, M. B., 2009, The Certified Quality Engineer Handbook.

7. DEPF (Direction des Etudes et des Prévisions Financières), 2013, Le secteur de transport des marchandises : Contraintes et opportunités.

8. DEPF (Direction des Etudes et des Prévisions Financières), 2016, Le transport maritime des marchandises au Maroc: Evolution et perspectives.

9. IIASA, 2017, International Institute for Applied Systems Analysis, http://www.iiasa.ac.at/.

10. Kammas, S., 2015, Pratiques de développement durable chez les prestataires logistiques marocains : quel levier de performance globale ?, Université Paul-Valery, Monpelier.

11. Landeta, J, 2006, Current validity of the Delphi method in social sciences, Technological Forecasting and Social Change, Vol.73, No.5, 467-482.

12. MET (Ministère d'Equipement et de transport), 2006, La Logistique du Commerce et la Compétitivité du Maroc.

13. Okoli, C., et Pawlowski, S., 2004, The Delphi method as a research tool: an example, design considerations and applications, Information \& Management, Vol. 42, pp. 15-29.

14. Pelet, J.E, 2011, Le e-commerce renforcé par les réseaux sociaux numériques : résultats d'une application expérimentale de la méthode 
de Delphi, Colloque de l'association Information \& Management(AIM), Saint- Denis, Ile de la Réunions.

15. PNAUD, 2015, Glogal Environmental Facility United Development Programme Country: Morocco Project Document.

16. Van Wulpen, C., 2014, Le secteur du transport et de la logistique au Maroc, Flanders Investment and Trade.

17. Vernette E., 1985, La méthode Delphi : potentiel pour l'identification des attributs déterminants; un pré-test, Actes du 1er colloque de l'Association Française de Marketing, pp. 169-191. 